\title{
Étude microtopographique des fortifications de terre de Haute-Normandie
}

Anne-Marie Flambard-Héricher, Bruno Lepeuple, Daniel Étienne, Gilles Deshayes, Sébastien Lefèvre, Jimmy Mouchard, Thomas Guérin et Aude Painchault

\section{OpenEdition} Journals

Édition électronique

URL : http://journals.openedition.org/adlfi/5262

ISSN : 2114-0502

Éditeur

Ministère de la culture

Référence électronique

Anne-Marie Flambard-Héricher, Bruno Lepeuple, Daniel Étienne, Gilles Deshayes, Sébastien Lefèvre, Jimmy Mouchard, Thomas Guérin et Aude Painchault, «Étude microtopographique des fortifications de terre de Haute-Normandie », ADLFI. Archéologie de la France - Informations [En ligne], HauteNormandie, mis en ligne le 01 mars 2006, consulté le 01 mai 2019. URL : http:// journals.openedition.org/adlfi/5262

Ce document a été généré automatiquement le 1 mai 2019.

(c) Ministère de la Culture et de la Communication, CNRS 


\title{
Étude microtopographique des fortifications de terre de Haute- Normandie
}

\author{
Anne-Marie Flambard-Héricher, Bruno Lepeuple, Daniel Étienne, Gilles \\ Deshayes, Sébastien Lefèvre, Jimmy Mouchard, Thomas Guérin et Aude \\ Painchault
}

Date de l'opération : 2006 (PC) ; 2006 (PI)

Inventeur(s) : Flambard-Hérichier Anne-Marie (ENS SUP) ; Lepeuple Bruno ; Etienne Daniel ; Deshayes Gilles (GAVS) ; Lefèvre Sébastien (SAR) ; Mouchard Jimmy (ENS SUP) ; Guérin Thomas ; Painchault Aude

1 L'objectif du groupe de travail qui s'est constitué à l'université de Rouen, au sein du GRHIS (EA 3831), avec l'appui du service régional de l'Archéologie de Haute-Normandie, sous la direction d'Anne-Marie Flambard-Héricher est de pratiquer de façon systématique des relevés microtopographiques de fortifications de terre comportant éventuellement des maçonneries, de les décrire suivant la grille élaborée par le projet collectif de recherche (PCR) développé en 1982 et de les interpréter à la lumière de cet examen approfondi, qui peut être croisé avec des observations de surface systématiques: notamment microreliefs, répartition des pierres taillées et mode de taille, répartition de la flore.

Dix sites de dimensions variables ont été traités au cours de l'année 2006. Ce sont: la motte de Cléry aux Andelys(Eure), Lyons-la-Forêt(Eure), les fortifications de Guerville (Seine-Maritime), Corneville (Eure), Melleville (Seine-Maritime), Angiens (Seine-Maritime), Hacqueville (Eure), Longchamps (Eure), de La Butte de l'Écuyer à Vatteville-la-Rue (Eure), et de La Butte au Diable à Maulévrier-Sainte-Gertrude (SeineMaritime). Ils seront présentés successivement dans les pages qui suivent par ordre alphabétique du nom des communes. (Responsable du PCR : FLAMBARD-HERICHER AnneMarie) 


\section{Andelys Les (Eure)}

\section{Cléry, Le Muret}

Le château de Cléry se situe sur le plateau, à $2 \mathrm{~km}$ au sud-est du célèbre château Gaillard. La fortification se présente comme une motte dont la plateforme sommitale de $20 \mathrm{~m}$ de diamètre culmine à $5 \mathrm{~m}$ au-dessus du plateau environnant. Les fossés, d'une largeur moyenne de $10 \mathrm{~m}$ pour $3 \mathrm{~m}$ de profondeur maximale, sont entièrement en eau. Sur trois de ses côtés, le site est entouré par des îlots bâtis qui ont gommé la trace d'une éventuelle basse-cour.

4 La plateforme de la motte est complexe et dispose de nombreux aménagements. Le quart nord-ouest est marqué par une large échancrure de forme approximativement carrée de $10 \mathrm{~m}$ de côté. Au fond de cette dépression se distinguent deux sondages anciens qui ont révélé des maçonneries faites de silex liés au mortier. Il s'agit de la trace vraisemblable d'une tour carrée liée à un mur parcourant la périphérie de la motte. Au centre du tertre s'ouvre une dépression de $5 \mathrm{~m}$ de profondeur, de plan rectangulaire de $0,90 \mathrm{~m} \times 0,70 \mathrm{~m}$. La régularité de la structure invite à l'interpréter comme un puits dont le cuvelage, complètement disparu, était en bois.

5 Les constructions maçonnées, postérieures à l'état primitif du site, peuvent être mises en relation avec la mention, dans les Grands Rôles de l'Échiquier de Normandie, en 1198, de travaux de réaménagement pour que le site serve de poste avancé au château Gaillard. Il s'agissait de compléter le dispositif défensif mis en place par Richard cœur de Lion. (Responsable de l'étude et du relevé : LEPEUPLE Bruno)

\section{Angiens (Seine-Maritime)}

\section{La Motte}

6 Le village d'Angiens est situé sur le plateau du pays de Caux, entre les vallées du Dun et de la Durdent. La motte, implantée au cœur du village actuel, est relativement bien conservée mais son éventuelle basse-cour a totalement disparu, vraisemblablement détruite par l'implantation d'un garage à l'est du site. La motte consiste en un tertre ovoïde de $15 \mathrm{~m}$ de diamètre sommital pour une élévation d'environ $4 \mathrm{~m}$; un fossé comblé, partiellement discernable, entourait toute ou partie de la motte.

7 Le village d'Angiens est mentionné pour la première fois en 1150 et un Guillaume, seigneur d'Angiens, est évoqué en 1180. La fortification est probablement érigée à cette date sans que l'on puisse être plus précis. Néanmoins, les troubles consécutifs à la mort du Conquérant pourraient être une période favorable à cette édification.

8 La motte connaît une réaffectation inhabituelle en 1943 lorsque l'occupant allemand décide de la construction, sous la motte, d'une chambre souterraine pour se protéger des bombardements alliés. Cette chambre, imparfaitement comblée, pourrait éventuellement participer à la dégradation du site (Responsable de l'étude et du relevé : ETIENNE Daniel). 


\section{Corneville-sur-Risle (Eure)}

\section{Le Bois Cany}

9 La fortification domine de plusieurs dizaines de mètres un vallon sec, axe de franchissement de la vallée de la Risle. De $45 \mathrm{~m}$ (nord-sud), sur $50 \mathrm{~m}$ (est-ouest) soit $2250 \mathrm{~m}^{2}$, la fortification est composée d'un tertre dessinant un quadrilatère irrégulier situé sur le rebord d'un large éperon dominant une cavée qui descend dans la vallée de la Risle. Ce tertre est entouré d'un fossé régulier, dont les terres semblent avoir été rejetées vers l'intérieur du site, ce qui lui confère un aspect cratériforme. Les fossés apparaissent surtout marqués sur trois côtés. Naturellement défendu par le relief accidenté et le versant, le quatrième côté se distingue plus ou moins sous la forme d'une petite « terrasse ». On note également la présence d'un petit talus au nord-est.

On ne dispose d'aucune source écrite mentionnant la fortification dont il demeure difficile de cerner la fonction. La morphologie assez particulière de ce site le différencie des autres fortifications de la région de la Risle. Il est néanmoins comparable à celles de Basse-Normandie (Orne) ou anglaises datées généralement des $\mathrm{XI}^{\mathrm{e}} \mathrm{s}$. et $\mathrm{XII}^{\mathrm{e}} \mathrm{s}$. La localisation de cette fortification laisse supposer qu'elle a pu constituer un ouvrage destiné à contrôler la voie de communication située au fond du vallon. En outre, située à la périphérie des terres dépendant des seigneurs de Pont-Audemer (Beaumont-Meulan), elle a pu servir à défendre les limites de leur domaine. De plus, la fortification du « Bois Cany » fait face à un autre site défensif situé à Appeville-Annebault, au lieu-dit « Le Vieux Montfort ", qui domine un autre axe de franchissement naturel de la vallée de la Risle et protège les limites des possessions des seigneurs de Montfort (-sur-Risle). (Responsables de l'étude et du relevé: DESHAYES Gilles, LEFEVRE Sébastien, MOUCHARD Jimmy).

\section{Guerville (Seine-Maritime)}

\section{Le Vieux Château}

11 Le village de Guerville est établi à l'ouest du plateau séparant les vallées de l'Yeres et la Bresle. L'érosion a créé un éperon qui, en plan, évoque un triangle isocèle. Construit sur la base de ce triangle, le château protège le plateau ensuite voué à l'agriculture. Si vers 1830 , on voyait encore quelques restes des murs d'enceinte et de tourelles semicirculaires, seuls subsistent aujourd'hui le tertre et une petite partie de la contrescarpe. Le parcellaire du village conserve néanmoins le plan de la basse-cour et des aménagements de l'enceinte villageoise.

12 Le tertre, entaillé au nord-ouest, présente l'aspect d'une plateforme circulaire d'environ $80 \mathrm{~m}$ de diamètre surplombant le relief extérieur de $2 \mathrm{~m}$ à $4 \mathrm{~m}$. Il était entouré d'un fossé, d'une profondeur d'au moins $5 \mathrm{~m}$, vraisemblablement en eau. Une surélévation sur le pourtour sud de la plateforme pourrait marquer la présence de bâtiments enfouis. Au nord du tertre, un fossé, réutilisé pour y placer une sente, matérialise le tracé de l'ancienne basse-cour en forme de croissant et enveloppant le tiers nord-est du site. L'actuelle rue de l'Église coupe la basse-cour en son milieu, témoignage de l'ancien 
chemin menant de l'église au château. L'enceinte du bourg est encore visible sur une partie de son tracé sud, le long de l'actuelle rue auxJuifs; son tracé nord s'est probablement conservé dans une sente reliant la basse-cour à la place de la Mairie.

Il s'agit d'une zone de défrichement isolant, à partir du milieu du $\mathrm{XI}^{\mathrm{e}} \mathrm{s}$., la haute forêt d'Eu de celle du Triage. La première mention date de 1130, quand l'archevêque de Rouen fait don d'une pièce de terre située dans une zone nouvellement essartée de la forêt deGuerville. Au milieu du XII ${ }^{\mathrm{s}}$., la terre deGuerville appartient à Raoul de Monchaux qui l'échange avec Henri I ${ }^{\text {er }}$ comte d'Eu, qui fait probablement construire le château, bien que la tradition attribue cette fondation à Jean $\mathrm{I}^{\mathrm{er}}$ de Brienne, comte d'Eu à la fin du XIII ${ }^{e} s$. Les comtes d'Eu vont y séjourner relativement souvent. Le village, vraisemblablement contemporain du château, compte alors cinquante parrochiani ; des portes sont mentionnées en 1240 et un moulin a vent est attesté en 1488. Le talus longeant l'actuelle rue du $1^{\text {er }}$ septembre, anciennement rue Bâtarde, pourrait indiquer un accroissement du bourg dans cette direction. Enfin, des vignes étaient encore cultivées sur la commune à l'extrême fin du XVIII ${ }^{\mathrm{e}} \mathrm{s}$. (Responsable de l'étude et du relevé : ETIENNE Daniel).

\section{Hacqueville (Eure)}

\section{Le Parc}

14 Le château d'Hacqueville, sur le plateau du Vexin, prend place sur les franges du village actuel, à $200 \mathrm{~m}$ de l'église paroissiale. Il est associé à une cour de ferme dont les bâtiments forment un ensemble clos. La fortification se présente sous la forme d'un tronc de cône large et peu élevé. La plateforme sommitale a un diamètre de $50 \mathrm{~m}$ et ne domine que de $3 \mathrm{~m}$ le plateau. Un large fossé, intégralement comblé depuis 1975, ceinturait l'ensemble $\mathrm{du}$ site, seuls quelques reliefs permettent d'estimer sa largeur à $25 \mathrm{~m}$ (Fig. $\mathrm{n}^{\circ} 1$ : Hacqueville : le Parc, plan et coupe du château).

La plateforme est occupée par deux bâtiments qui prennent appui sur des murs anciens témoins d'une enceinte de pierre polygonale aveugle, non contrefortée, faite de silex et de pierres calcaires équarries pour les chaînages d'angle. Sous le bâtiment occidental ont été aménagées quatre caves dont deux sont médiévales. La première construite en fosse est constituée d'une salle voûtée en berceau de 5,4 m x 3,3 m. Dans le mur nord s'ouvre une porte qui permet d'accéder à la seconde cave faite d'un escalier droit et d'une galerie coudée desservant cinq cellules de stockage.

16 Les divers modes de construction et la datation des différentes parties, XIII ${ }^{e}$ s.-XIV ${ }^{e}$ s. et $\mathrm{XIV}^{\mathrm{e}} \mathrm{s}$. (étude réalisée par Deshayes Gilles) permettent de poser des hypothèses relatives à l'évolution du site. Une enceinte circulaire primitive pourrait avoir été comblée lors de l'aménagement de la première cave. C'est à cette époque qu'a été édifié le mur périphérique. Ces témoins ne sont que les vestiges d'un château plus puissant où étaient encore visibles, en 1639, un donjon et un pont-levis. La première mention du site, en 1144, est certainement largement postérieure à la naissance de la fortification. Le site primitif est à rapprocher d'un type d'ouvrages de terre de la première moitié du XI $\mathrm{s}$. que l'on rencontre au centre des domaines féodaux ce qui s'accorde avec les données de la géographie féodale : les terres d'Hacqueville relèvent de la seigneurie de Tosny, puissante 
famille normande à cette période. (Responsables de l'étude et du relevé : DESHAYES Gilles et LEPEUPLE Bruno).

\section{Longchamps (Eure)}

\section{Le Prieuré}

17 Le château de Longchamps se situe non loin des franges du massif forestier de Lyons. Il est installé au nord du village, au niveau d'une rupture de pente au-dessus d'un petit vallon sec. La fortification dessine un tronc de cône de $50 \mathrm{~m}$ de diamètre à la base pour $35 \mathrm{~m}$ au sommet, elle est partiellement isolée du flanc de coteau par un fossé de plus de $5 \mathrm{~m}$ de profondeur pour $25 \mathrm{~m}$ de largeur. Le tertre n'a pas fait l'objet d'un remblaiement significatif, les terres extraites ont été employées pour établir un fort rempart de terre visible dans le quart septentrional du site. Vers le nord-ouest, une terrasse de $40 \mathrm{~m}$ x $30 \mathrm{~m}$ est délimitée par un fossé dont une partie a disparu. Il est difficile d'y voir une basse-cour (celle-ci devait se développer vers le sud, occupé par une ferme), il s'agit plutôt d'un ouvrage avancé orienté vers le plateau.

Sur le pourtour de l'enceinte principale se développe un mur irrégulièrement conservé flanqué de cinq tours rectangulaires. Au centre se dressent les vestiges d'une tour circulaire dont il ne reste que le blocage. Le mur annulaire devait adopter un tracé fermé. Son élévation est percée de plusieurs ouvertures permettant l'accès au second niveau des tours et de deux baies qui indiquent la présence de bâtiments adossés. Les flanquements postérieurs au mur annulaire, adoptent tous le même plan. Leur élévation est faite de silex alterné avec des lignes de grès. L'espace intérieur est divisé en deux niveaux de tir avec l'aménagement d'archères à niche, deux disposées frontalement, puis trois sur chacun des côtés à l'étage. Au centre, la tour circulaire présente une forte épaisseur des murs, 3,50 m, et un faible espace intérieur, $3,70 \mathrm{~m}$ de diamètre. La seule fonction qui lui est assignable est la surveillance, l'exiguïté du volume interne interdit d'y voir un espace résidentiel. Celui-ci était plutôt distribué le long du mur annulaire, à la manière d'un shell keep.

Les Comptes de l'Échiquier de Normandie, en 1180, précisent que le roi d'Angleterre, Henri II Plantagenêt, est entré en possession du château. Le mur annulaire posé sur le sol géologique semble être bâti dès l'origine. Le fossé et les terres qui en sont issues, disposées en arc, sont logiquement présentes dans la première phase. L'ouvrage avancé, qui coupe nettement cette levée de terre, est le témoin d'une seconde phase de terrassements. Par les matériaux utilisés, les tours de flanquement, s'apparentent à une construction de la fin du Moyen Âge. En effet, le site a fait l'objet d'incessants travaux sous Philippe le Bel et ses successeurs jusqu'au début du XV $\mathrm{e}$. Préalablement, une phase de travaux commandée par les Plantagenêts à la fin du XII ${ }^{e}$ s. est probable. (Responsable de l'étude et du relevé : LEPEUPLE Bruno). 


\section{Lyons-la-Forêt (Eure)}

\section{Le Château} jonction de vallées drainées. Son emplacement à la lisière de la forêt domaniale du TraitMaulévrier, lui confère une position dominante sur la ville de Caudebec-en-Caux et sur la Seine.

Localisé au centre même du bourg de Lyons, le site est caractérisé par une très forte densité du bâti et un important morcellement parcellaire se superposant à l'ancien espace castral. Le cœur de celui-ci, légèrement ovale, est composé d'une grande plateforme d'environ $70 \mathrm{~m}$ dans l'axe nord-sud et $60 \mathrm{~m}$ dans l'axe est-ouest. Il n'y a sans doute pas de surélévation marquée du terrain, les terres extraites ont été rejetées vers le nord-ouest, pour prolonger la plateforme. C'est de ce coté que la dénivellation est la plus conséquente: environ $10 \mathrm{~m}$. Dans le quart sud-ouest du bourg, la topographie et le parcellaire permettent d'identifier un vaste espace de 3,3 ha, partiellement entouré d'un fossé formant un demi-cercle et raccordé au château sur son flanc occidental.

C'est à la périphérie de la plateforme que s'observent les principaux vestiges de maçonnerie. À l'extérieur de ce périmètre, d'autres sections de murs sont accolées à des habitations dans la moitié ouest du bourg. Le relevé des ruines permet de restituer une enceinte flanquée de sept tours, dont l'une, vers l'est, faisait fonction de porte. Son passage voûté est actuellement presque enterré. L'important remblaiement de l'intérieur du site permet d'envisager l'hypothèse d'une levée de terre périphérique, au moins dans la partie sud, face à la pente naturelle du coteau. À l'intérieur de la plateforme, une fouille privée effectuée entre 1979 et 1983 a mis au jour l'angle d'un bâtiment caractérisé par la présence de deux contreforts plats et d'un puits. Le mobilier céramique recueilli, non stratifié, fournit des indices quant à l'occupation du site entre le XII ${ }^{e}$ s. et le XVe $s$.

L'origine du château n'est pas clairement donnée par les textes. La première mention est de 1106, au début du règne d'Henri I ${ }^{\text {er }}$ Beauclerc, mais le site est certainement antérieur. Les phases maçonnées sont hétérogènes. N'étant pas, pour la plupart, liées entre elles, il n'est pas possible d'établir une chronologie relative. Les observations réalisées tant sur les plans que sur les matériaux, permettent d'affirmer qu'une grande partie de l'enceinte a été remaniée postérieurement au XIII ${ }^{\mathrm{e}}$ s., sans doute à la suite du grand incendie de 1299-1300. Seul le bâtiment dégagé lors de la fouille présente des caractères romans. Une reprise des investigations archéologiques prévue pour juin 2007 sur ce secteur permettra de préciser la fonction et la date de construction du bâtiment.

Au-delà du site fortifié, l'étude parcellaire a permis de reconnaître la présence d'un bourg castral fait de deux îlots bâtis et d'une place de marché toujours marquée par la présence d'une halle. (Responsable de l'étude et du relevé : LEPEUPLE Bruno).

\section{Maulévrier-Sainte-Gertrude (Seine-Maritime)}

\section{La Butte au Diable}

4 Le château de la Butte au Diable se situe en rebord du plateau du pays de Caux, à la 

D'orientation générale nord-ouest-sud-est, la structure comprend une haute-cour flanquée au sud-est, d'un donjon quadrangulaire de $18 \mathrm{~m}$ de côté sur le tracé de la courtine. Au nord-ouest et à l'ouest deux probables tours de flanquement, reliées par un passage, assuraient la protection face à la vallée. À l'est, munie de deux tours circulaires, l'entrée de la haute-cour appuyait la défense. Elle donnait accès à la basse-cour du château qui s'étend encore aujourd'hui jusqu'aux pieds de l'église du village dédiée à saint Léonard. Un profond fossé, encore très bien conservé, isole la haute-cour du reste du terroir ; deux murs le traversent au nord et au sud-est et relient le talus de la bassecour au reste de la fortification. Un remblai aménagé dans le fossé entre l'entrée de la haute-cour et la basse-cour a sans doute permis de remplacer un probable pont-levis. À l'intérieur de la haute-cour quelques reliefs révélés par les relevés semblent correspondre à des bâtiments résidentiels disposés tout le long du mur de courtine, autour du puits central. du XII ${ }^{e}$ s., même s'il est probable qu'une forteresse primitive existait déjà sur ce site occupé depuis l'époque gallo-romaine. Plusieurs phases de construction ont été mises en évidence. La première, peut remonter à l'époque des comtes d'Évreux probables commanditaires du château. Au XIVe s., sous la coupe des comtes de Savoie et en raison des nécessités de la guerre de Cent Ans, les tours de flanquement et les murs dans le fossé viennent renforcer la courtine et appuyer l'effet protecteur du donjon et du fossé. Le château est abandonné avant le $\mathrm{XVI}^{\mathrm{e}} \mathrm{s}$. et ses pierres ont servi de carrière pour les habitants du village. La seigneurie perdure quant à elle jusqu'au XVIII ${ }^{e}$ s., détenue successivement par deux familles prestigieuses de Normandie, les De Brézé et les Dufay du Taillis. (Responsables de l'étude et du relevé : GUERIN Thomas, PAINCHAULT Aude).

\section{Melleville (Seine-Maritime)}

\section{La Motte}

Le commune de Melleville est établie à l'ouest du plateau séparant les vallées de l'Yères et de la Bresle. La motte est implantée face à l'église paroissiale, dont elle est séparée par la route D 315 qui entaille le flanc est du site. Le tertre est aujourd'hui profondément mutilé ; un fossé comblé est encore partiellement discernable mais l'éventuelle bassecour a totalement disparu et aucun indice n'indique sa localisation. La motte de plan rectangulaire $(25 \mathrm{~m} \times 20 \mathrm{~m})$ a une élévation d'environ $4 \mathrm{~m}$. Des débris de brique moderne semblent indiquer une réaffectation du site après son abandon.

Mentionné en 1059, le village de Melleville est issu du mouvement d'essartage qui, au cours du XIe s., isole la haute forêt d'Eu de celle du Triage. À cette date est mentionné un seigneur de Melleville, Eugidius; plusieurs autres seigneurs de Melleville apparaissent dans le cartulaire du Tréport dont un Robert, vassal des comtes d'Eu, qui tient aussi le fief de Bully près de Neufchâtel-en-Bray. 
30 La date de fondation exacte n'est pas connue mais on peut envisager que la motte fut édifiée lors des périodes de troubles du XII ${ }^{e}$ s., soit pendant la crise de succession de Guillaume le Conquérant, soit pendant la période d'opposition entre Étienne et Henri Plantagenêt. Volontairement retaillée, la motte a du être réutilisée soit comme jardin d'agrément au XIX $\mathrm{s}$. soit pour servir de base à un éventuel moulin à vent évoqué au voisinage de l'église au XVII ${ }^{e}$ s. (Responsable de l'étude et du relevé : ETIENNE Daniel).

\section{Vatteville-la-Rue (Seine-Maritime)}

\section{La Butte de l'Écuyer}

31 Aujourd'hui isolée à l'entrée de la forêt de Brotonne (possession des Beaumont-Meulan), la fortification de La Butte de l'Écuyer est installée à proximité du côté ouest du méandre de la Seine. Malgré les nombreuses études archéologiques et historiques, le site n'a jamais fait l'objet d'un relevé topographique. Cette petite motte tronconique, construite avec des matériaux locaux (terre, graviers, silex, sablon) est assez érodée sur son pourtour. La partie ouest du tertre, sectionnée sur un rayon d'environ $3 \mathrm{~m}$ (lors de la construction de la D 65), offre une coupe franche. Le diamètre de la plateforme est d'environ $13 \mathrm{~m}$. Le fossé au tracé régulier, entoure aux trois-quarts la base du tertre. Il présente un profil en « $\mathrm{U}$ » et une profondeur moyenne de $1,10 \mathrm{~m}$.

L'ouvrage a été installé au voisinage d'un chemin médiéval, connu sous le nom de «Chemin du Roi » qu'emprunta Guillaume le Bâtard lorsqu'il se rendit à Arques après avoir traversé la Seine à Caudebec. Il reprend en partie l'ancien tracé d'une voie antique reliant Pont-Audemer à Caudebec. Il est possible que la fortification n'ait jamais été pourvue de fossé du côté du chemin qui semble se connecter à un autre plus petit, légèrement perpendiculaire, qui permet d'accéder à l'ancienne grève de la Seine. Peu de sites permettaient un tel contrôle de la rive de la boucle de Brotonne, à proximité du château des Meulan situé plus au nord. La Butte de l'Ecuyer se situait probablement à environ $25 \mathrm{~m} \mathrm{du}$ fleuve au Moyen Âge. Les dépôts alluvionnaires des Temps Modernes (XVII ${ }^{\mathrm{s}}$.) et l'endiguement de la Seine (XIX ${ }^{\mathrm{e}} \mathrm{s}$ ) ont provoqué le recul de la berge, fossilisant l'ancienne grève estuarienne.

Plusieurs hypothèses ont été avancées quant à la fonction du site : il peut s'agir soit d'un ouvrage avancé du château des Beaumont-Meulan sis à Vatteville-la-Rue (XII ${ }^{e}$ s.), soit d'un contre-château érigé par Henri $I^{\text {er }}$ Beauclerc lors du siège de ce même château en 1123-1124. D'un point de vue archéologique, en l'absence de fouilles, les deux hypothèses sont recevables. Aucune construction antérieure ou postérieure n'a été remarquée sur le site. Néanmoins, un fragment de mortier gallo-romain (Bas-Empire) a été mis au jour en septembre 2005 lors d'un rafraîchissement des coupes des sondages effectués par L. Charlier en 1838. (Responsables de l'étude et du relevé : DESHAYES Gilles, MOUCHARD Jimmy).

FLAMBARD-HERICHER Anne-Marie 


\section{ANNEXES}

Fig. $\mathrm{n}^{\circ} 1$ : Hacqueville : le Parc, plan et coupe du château

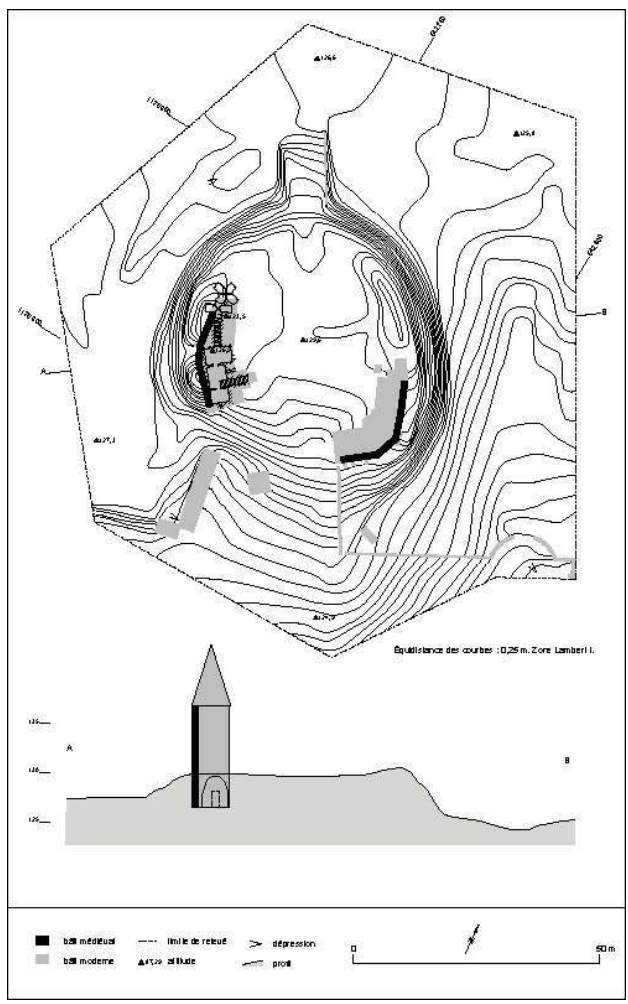

Auteur(s) : Deshayes, Gilles ; Guérin, T. ; Vincent, J.-B. ; Lepeuple, Bruno. Crédits : ADLFI (2006)

\section{INDEX}

Index chronologique : Temps Modernes, XIe siècle apr. J.-C., XIIe siècle apr. J.-C., XIIIe siècle apr. J.-C., XIVe siècle apr. J.-C.

operation Projet collectif de recherche (PCR), Prospection inventaire (PI)

\section{AUTEURS}

\section{ANNE-MARIE FLAMBARD-HÉRICHER}

ENS SUP

\section{GILLES DESHAYES}

GAVS

SÉBASTIEN LEFÈVRE

SAR 
JIMMY MOUCHARD

ENS SUP 